New Understanding of Poloidal Rotation

Measurements in a Tokamak Plasma

by

R.E. Bell and E.J. Synakowski

May 2000

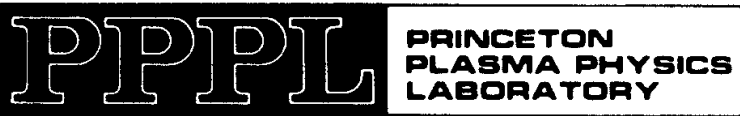

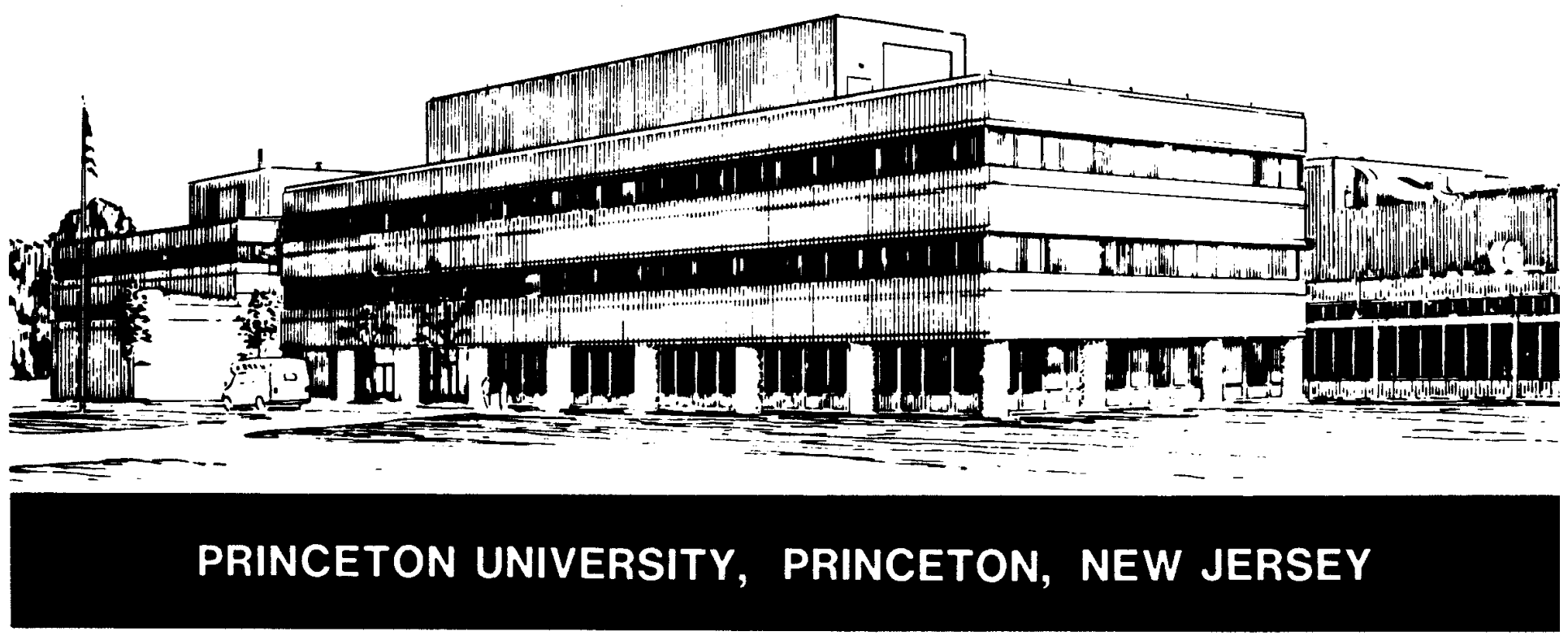




\section{PPPL Reports Disclaimer}

This report was prepared as an account of work sponsored by an agency of the United States Government. Neither the United States Government nor any agency thereof, nor any of their employees, makes any warranty, express or implied, or assumes any legal liability or responsibility for the accuracy, completeness, or usefulness of any information, apparatus, product, or process disclosed, or represents that its use would not infringe privately owned rights. Reference herein to any specific commercial product, process, or service by trade name, trademark, manufacturer, or otherwise, does not necessarily constitute or imply its endorsement, recommendation, or favoring by the United States Government or any agency thereof. The views and opinions of authors expressed herein do not necessarily state or reflect those of the United States Government or any agency thereof.

\section{Availability}

This report is posted on the U.S. Department of Energy's Princeton Plasma Physics Laboratory Publications and Reports web site in Calendar Year 2000. The home page for PPPL Reports and Publications is: http://www.pppl.gov/pub_report/

DOE and DOE Contractors can obtain copies of this report from:

U.S. Department of Energy

Office of Scientific and Technical Information

DOE Technical Information Services (DTIS)

P.O. Box 62

Oak Ridge, TN 37831

Telephone: (865) 576-8401

Fax: (865) 576-5728

Email: reports@adonis.osti.gov

This report is available to the general public from:

National Technical Information Service

U.S. Department of Commerce

5285 Port Royal Road

Springfield, VA 22161

Telephone: $1-800-553-6847$ or

(703) $605-6000$

Fax: (703) 321-8547

Internet: http://www.ntis.gov/ordering.htm 


\title{
New Understanding of Poloidal Rotation Measurements in a Tokamak Plasma
}

\author{
R. E. Bell, E. J. Synakowski \\ Princeton Plasma Physics Laboratory \\ Princeton NJ, 08543
}

\begin{abstract}
Significant atomic physics corrections to the measured poloidal velocity using charge exchange spectroscopy have been neglected when interpreting impurity poloidal velocity. In the presence of a magnetic field, the gyro motion of the impurity ion along with the finite lifetime of the observed state results in an additional line shift that scales with ion temperature and magnetic field strength. Effects of collisions on the lifetime of excited states, cascades from higher longer-lived $n$ levels, and charge exchange from excited beam neutrals are calculated to determine necessary corrections to the measured poloidal velocity. The accuracy of computed corrections is tested utilizing symmetric upward and downward views from the TFTR poloidal rotation diagnostic.
\end{abstract}

\section{INTRODUCTION}

Charge exchange spectroscopy has been used as a tool to diagnose plasma parameters for over two decades ${ }^{1}$. High energy neutral beams serve as effective probes penetrating deep into the core of high temperature plasmas, where light ions are otherwise fully ionized, thereby making spectroscopic measurements useful. Localized measurements of plasma profiles of ion temperature, $T_{i}$, and toroidal velocity, $v_{\phi}$, have routinely been made on most tokamaks with neutral beams. Measurements of poloidal velocity, $v_{\theta}$, have been applied to the plasma edge to measure changes in the radial electric field ${ }^{2}$ which is associated with the formation of a transport barrier and the suppression of turbulence. More recently, $v_{\theta}$ measurements have been directed to the plasma core, ${ }^{3}$ to investigate core transport barriers and to evaluate the radial electric field. The radial electric field can be measured indirectly using the radial force balance equation,

$$
E_{r}=\frac{1}{e Z n} \frac{\partial p}{\partial r}+v_{\phi} B_{\theta}-v_{\theta} B_{\phi}
$$

where the impurity ion pressure $p$, ion density, $n, \mathrm{t} v_{\phi}$, and $v_{\theta}$, are all determined from charge exchange spectroscopy.

Proper interpretation of the measurements requires consideration of many details to correctly determine the plasma parameters of interest. Measurement of $T_{i}$ involves the measurement of the Doppler width of a Maxwellian distribution of ions; determination of the plasma flow involves the measurement of a line shift. The charge exchange process distorts the velocity distribution of the ions due to a charge exchange cross section that depends on the collision energy between the incoming beam neutral and the impurity ion. ${ }^{4,5}$ This changes the apparent $T_{i}$ and results in extraneous velocity shifts, not associated with plasma motion, that scale with $T_{i}$. Knowledge of the charge exchange cross section is necessary to properly compute corrections to the measured velocity and temperature. Molecular hydrogen in the beam source results in three 
energies of fast neutrals at the full, half, and third energy of the beam voltage. As the beam neutrals penetrate to the plasma core, the energy components are ionized at different rates, changing their relative populations, so a beam attenuation calculation is required to determine local densities of beam neutrals. A small fraction of the incoming beam neutrals are in excited states that have much larger charge exchange cross sections with the impurity ions than ground state neutrals, and are able to influence the reaction rate at low collision velocities. ${ }^{6,7}$ The neutral sources typically used on tokamaks are intended for heating the plasma and so have large footprints. The beam width, however, remains small compared to the major radius of the tokamak, making good radial resolution possible for toroidal views with the proper choice of viewing location. The vertical size of neutral sources, though, is large compared to the minor radius particularly as the plasma center is approached yielding chord averaged measurements for vertical views that must be inverted to obtain local velocity values. ${ }^{8}$

Considering the above details, a poloidal rotation diagnostic was installed on the Tokamak Fusion Test Reactor (TFTR) to measure poloidal flow. ${ }^{9}$ Unexpected poloidal velocities were measured in a variety of discharges that contradicted neoclassical predictions. ${ }^{3}$ The magnitude and direction of the measured poloidal velocity made it difficult to explain with known processes. Some of these measurements, namely a large transient poloidal velocity precursor to an enhanced confinement mode, were confirmed by independent measurements. ${ }^{10}$ The observation that the measured poloidal flow was roughly proportional to $T_{i}$ over a wide range of plasma parameters seemed to link the results. This last observation lead to the realization that charge exchange spectroscopic measurements in the plane of the gyro orbit of the impurity ions suffered an additional complication arising from the motion of the ion in its gyro orbit and the finite lifetime of the observed state. ${ }^{11}$ In essence, the treatment of the impurity ion motion as a fluid flow was inadequate when viewing perpendicular to the magnetic field where particle kinetics are important. Views perpendicular to the direction of the neutral beam were previously considered immune from the effects of the energy dependent charge exchange cross section. This is not true when viewing in the plane of the gyro motion of the ions, as explained below.

Gyro motion and finite lifetime effects on velocity measurements have been considered previously, where a pseudo velocity was produced by the spatial variation of excitations rates. ${ }^{12}$ Quite recently, a derivation of the changes in the distribution function of ions after undergoing charge exchange treated the gyro orbit and finite lifetime effects on the line shape. ${ }^{13}$

The goal of this paper is to examine the relevant atomic physics affecting the interpretation of the poloidal velocity measurements so the pseudo velocity associated with gyro motion of the ion can be calculated. Previous work has addressed the factors affecting the total emission from impurity ions after the charge exchange process to properly evaluate ion densities in the plasma. ${ }^{14}$ In this study, we demonstrate that it is crucial to consider atomic physics effects beyond the energy-dependent charge exchange processes normally considered in evaluating ion densities. In particular, the timedependent details of the decay processes themselves must be included to properly correct for the spectral line shifts not induced by plasma rotation. The measurements from TFTR serve to check the accuracy of the calculated values. Symmetric opposing sightlines used in the TFTR poloidal rotation diagnostic allow us to isolate the effects of the gyro motion and finite lifetime from the actual poloidal velocity.

This paper is organized as follows. Section 2 summarizes the charge exchange process and the line shifts due to the charge exchange cross section. Section 3 presents a mathematical framework for the gyro-motion and finite-lifetime effects. Section 4 reexamines the experimental data to gain insights into the accuracy of the calculated lifetimes and the cross sections. Section 5 presents a more complete treatment with a 
collisional-radiative model to determine corrections. In Section 6, an evaluation of the effective lifetime of the observed level is presented.

\section{ENERGY DEPENDENT CHARGE EXCHANGE PROCESS}

The reaction for electron capture by a fully ionized carbon impurity ion from a deuterium beam neutral is

$$
D^{0}\left(n_{D}\right)+C^{6+} \rightarrow D^{+}+C^{5+}(n)
$$

where the beam neutral is usually in the ground state $\left(n_{D}=1\right)$, but can also be excited $\left(n_{D}=2,3, \ldots\right)$, and the product hydrogenic impurity ion is in an excited state $(n)$. The levels most likely to be populated are near $n \approx n_{D} Z^{3 / 4} .5$ Here, we will be concerned with the emission from the $n=8-7$ transition of $C^{5+}$ at $5290.5 \AA$. The emission from a unit volume of plasma is proportional to the product of the beam neutral density, the impurity ion density, and the charge exchange rate coefficient,

$$
E \propto n_{b} n_{i}\langle\sigma v\rangle^{C X}
$$

with

$$
\langle\sigma v\rangle^{C X}=\iiint d v^{3} \sigma^{C X}\left|\vec{v}-\vec{v}_{b}\right| f\left(\vec{v}-\vec{v}_{r o t}\right),
$$

where $\sigma^{C X}=\sigma^{C X}\left(\left|\vec{v}-\vec{v}_{b}\right|\right)$ is the energy dependent charge exchange cross section, $\left|\vec{v}-\vec{v}_{b}\right|$ is the collision velocity between the impurity ion and the neutral, $f(v)$ is the Maxwellian velocity distribution of the fully ionized impurity ion, and $\vec{v}_{\text {rot }}$ is the rotational velocity of the impurity ion.

To illustrate how the energy dependent cross section can cause a non-motional line shift, we consider a one dimensional system in which the plasma is viewed in the direction of a deuterium neutral beam. The impurity ions in the distribution function moving away from the viewer are also moving away from the neutral beam and will have a lower collision energy with the beam neutrals than ions moving toward the beam and the viewer. The charge exchange cross section for $\mathrm{C}^{6+}$ increases with collision velocity as shown in Figure 1. Impurity ions moving toward the viewer (blue shifted from the line center) are more likely to undergo charge exchange than ions moving away (red shifted). With the blue side of the line enhanced and the red side diminished, an apparent shift in the emission line will be observed without plasma motion of the parent ion. Each of the three energy components of the deuterium beam will sample different portions of the charge exchange cross section. The higher the temperature, the more of the cross section that is sampled, and the larger the apparent shift. The observed emission from the hydrogenic product ion $\left(\mathrm{C}^{5+}\right)$ will be the sum of the three components with a line position that is shifted from the distribution of $\mathrm{C}^{6+}$.

The situation in the plasma requires an integral over velocity space in three dimensions, but the result is the same. If the view of the plasma has a component in the direction of the neutral beam, there will be an extra line shift (shifted toward the neutral beam) that is not consistent with plasma motion of the parent ion. For the observed state, there is a net current toward the neutral beam, since charge exchanged is favored for ions moving toward the beam. The extraneous line shift scales with $T_{i}$ and is strongly view dependent. This effect will be minimized when viewing perpendicular to the neutral beam. 

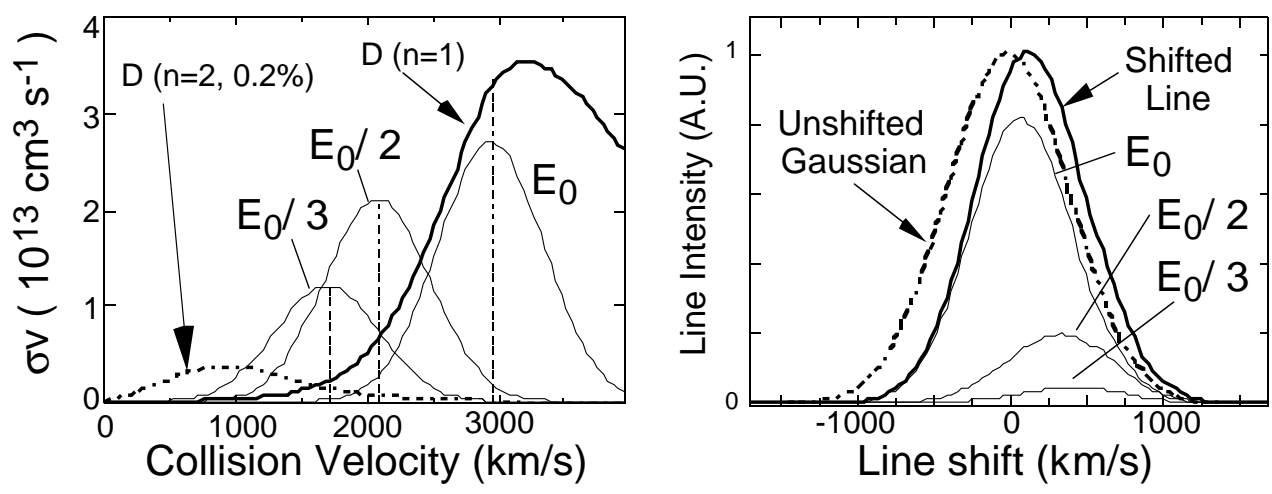

Figure 1. A 1D example of a line shift due to an energy dependent charge exchange cross section. (a) The distribution function for the three energy components of the neutral beam $\left(\mathrm{E}_{0}, \mathrm{E}_{0} / 2, \mathrm{E}_{0} / 3\right)$ are overlaid with the charge exchange cross section for ground state deuterium neutrals. Also shown is the relative cross section for a relative population of exited neutrals with $n=2$, which are important for low collision velocities. (b) The distributions for each of the energy components is shifted due to enhanced emission for ions moving toward the beam and diminished emission for ions moving away from the beam. The sum of the three components gives the observed line shift in this 1D example.

\section{GYRO MOTION AND FINITE LIFETIME EFFECTS}

Single particle motion in the presence of a magnetic field can significantly alter the velocity distribution of ions having undergone charge exchange with directed beam neutrals. If there is a component of the neutral beam velocity in the plane of the gyro orbit, there will be a net velocity in the direction of the neutral beam that scales with $T_{i}$. If the product ion does not radiate immediately, the gyro motion will redirect some of this net velocity vertically. The situation is shown schematically in Fig. 2 where the neutral beam is directed radially inward and the gyro motion is clockwise. There will be a larger collision velocity with beam neutrals at the top of the gyro orbit than at the bottom, causing a net radial velocity. If the lifetime of the upper level of the observed state is $\tau$, then the ions will, on average, precess $\omega \tau$ before radiating, where $\omega$ is the

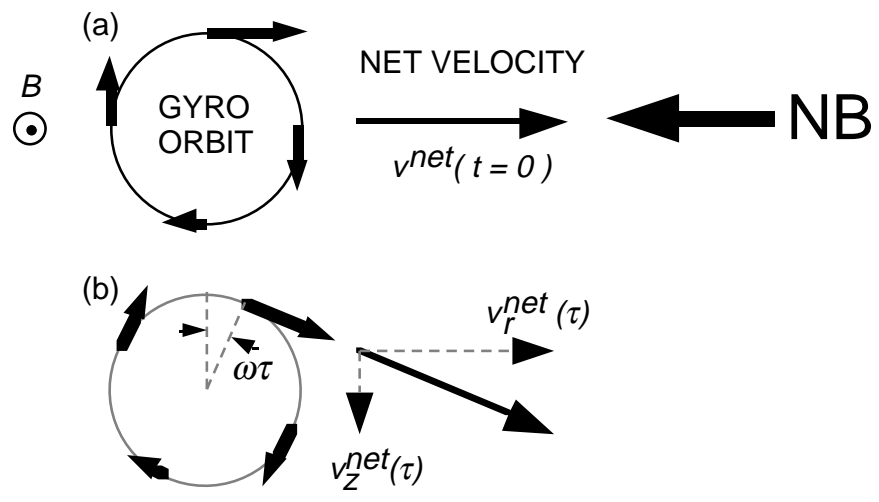

Figure 2. Impurity ions gyrate around magnetic field lines and have collision energies with beam neutrals that vary with gyro angle. (a) A larger collision energy at the top of the gyro orbit produces more product ions $\left(\mathrm{C}^{5+}\right)$ from charge exchange than at the bottom of the orbit causing a net velocity toward the neutral beam. (b) After a time $\tau$, the asymmetry has precessed $\omega \tau$, where $\omega$ is the ion gyrofrequency, resulting in a net downward velocity independent of poloidal velocity. 
(a)

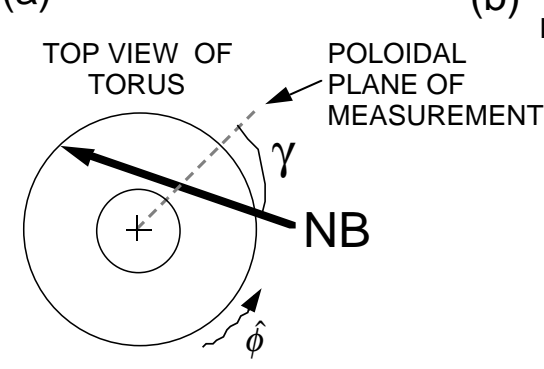

(b)

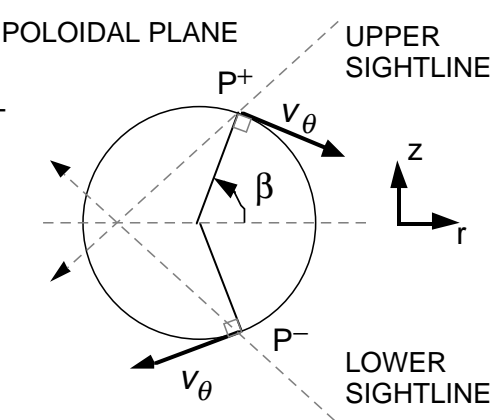

(c)

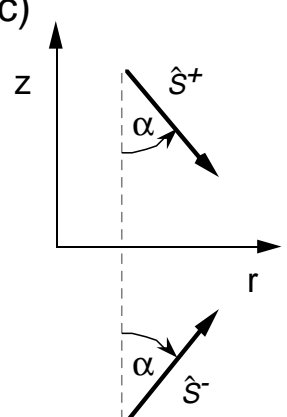

Figure 3. TFTR viewing geometry. (a) The angle $\gamma$ is the angle between the neutral beam direction and the plane of measurement. (b) Symmetric up/down views sample locations mirrored about the midplane. The angle $\beta$ is used to define the direction of the poloidal velocity. (c) The angle $\alpha$ is the angle of each sightline from vertical. $\hat{s}^{ \pm}$are unit vectors in the viewing direction for the top/bottom sightlines.

cyclotron frequency, causing a net downward vertical velocity not associated with plasma motion. The cyclotron frequency is given by $\omega=e Z B / M=9.64 \times 10^{7} Z B / \mu$, where $Z$ is the ion charge, $B$ is the magnetic field in Tesla, $\mu$ is the ion mass in atomic units. The typical lifetime for emission lines in the visible is $\approx 10^{-9} \mathrm{sec}$. For $\mathrm{C}^{5+}$ with $Z=5$ and $\mu=12$, and typical tokamak magnetic fields $B_{\phi}=2-5 \mathrm{~T}, \omega \tau \approx 0.1-$ 0.25 , i.e. $5^{\circ}-15^{\circ}$. Depending on $T_{i}$, the radial component of this pseudo velocity can be quite large compared to actual poloidal velocity. The gyro motion induced vertical component will scale with both $T_{i}$ and $B_{\phi}$.

Previous treatments of the effects of the charge exchange cross section have neglected this particle motion. This was acceptable for toroidal views which were normal to the plane of gyro motion, and also for poloidal views at the colder plasma edge where the effect was less pronounced. For hot interior plasmas and typical magnetic fields, it is important to correct for this effect that can dominate the actual poloidal flow.

We will define a mathematical framework to understand this contribution to poloidal velocity measurements of $\mathrm{C}^{5+}$ to recover the actual poloidal velocity of $\mathrm{C}^{6+}$. We will consider the TFTR geometry. The TFTR neutral beams were tangentially oriented both co-directed and counter-directed with respect to the plasma current, allowing control over momentum input. Three neutral beam sources were viewed by the poloidal rotation diagnostic. Figure 3a shows the toroidal direction, $\hat{\phi}$, counter clockwise from a top view of the torus, and $\gamma$ is defined as the angle between the plane of the poloidal velocity measurements and the neutral beam direction. Symmetric up/down views of the plasma sampled identical locations above and below the midplane in a strictly poloidal plane, i.e. no toroidal components. Figure $3 b$ shows upper and lower sightlines in the plane of measurement, where the upper sightline intersects point $\mathrm{P}^{+}$above the midplane, while the corresponding lower sightline intersects point $\mathrm{P}^{-}$below the midplane; note that the radial component of the poloidal velocity at these two points is equal and opposite. The angle $\beta$ is used to define the direction of the poloidal velocity at any point (see Fig. 3b). The angle of each sightline from vertical is designated $\alpha$ (Fig. 3c). The direction of the plasma current is $\hat{\phi}$, opposite to the toroidal magnetic field direction . The gyro angle, $\theta_{g}$, is defined from the radial axis.

Extending Equations (2-3) to include all beam component and beam sources and explicitly adding an integral over time, the measured poloidal velocity can be defined as

$$
\vec{v}_{a p p}=\frac{\sum_{j} \sum_{k} \iiint d v^{3} \int \mathrm{dt} \mathrm{e}^{-\mathrm{t} / \tau} n_{j k}^{b} n^{i} \sigma_{j k}^{C X}\left|\vec{v}-\vec{v}_{j k}^{b}\right| f\left(\vec{v}-\vec{v}_{r o t}\right) \vec{v}^{C X}(t)}{\sum_{j} \sum_{k} \iiint d v^{3} \int \mathrm{dt} \mathrm{e}^{-\mathrm{t} / \tau} n_{j k}^{b} n^{i} \sigma_{j k}^{C X}\left|\vec{v}-\vec{v}_{j k}^{b}\right| f\left(\vec{v}-\vec{v}_{r o t}\right)}
$$


where the subscripts $j, k$ correspond to the indices of the beam energy components and beam sources, respectively. Here we consider only the upper level of the observed transition with a lifetime $\tau$. The denominator is the measured intensity. The numerator is the intensity weighted by the time dependent velocity, $\vec{v}^{C X}(t)$. This is the velocity of the product ion $\left(\mathrm{C}^{5+}\right)$, including thermal velocity with gyro motion, and both toroidal and poloidal rotation,

$$
\begin{gathered}
\vec{v}^{C X}=\vec{v}_{t h}+\vec{v}_{\phi}+\vec{v}_{\theta}, \\
\vec{v}_{t h}=v_{\|} \hat{\phi}+v_{\perp} \sin \left(\theta_{g}-\omega t\right) \hat{r}-v_{\perp} \cos \left(\theta_{g}-\omega t\right) \hat{z}
\end{gathered}
$$

and $v_{\|}^{2}+v_{\perp}^{2}=v_{t h}^{2}=2 T_{i} / M_{i}$, where $v_{t h}$ is the thermal velocity and $M_{i}$ is the impurity ion mass. The toroidal velocity, $\vec{v}_{\phi}=v_{\phi} \hat{\phi}$, can be comparable in magnitude to the beam velocity. The poloidal velocity term depends on whether the upper sightline or lower sightline is used for the measurement (refer to Fig. 3b),

$$
\vec{v}_{\theta}^{ \pm}= \pm v_{\theta} \sin \beta \hat{r}-v_{\theta} \cos \beta \hat{z}
$$

with the superscript "+" indicating the upper sightline and "-" indicating the lower sightline. The neutral beam velocity is given by,

$$
\vec{v}_{j k}^{b}=v_{j k}^{b}\left(\sin \gamma_{k} \hat{\phi}-\cos \gamma_{k} \hat{r}\right), \quad v_{j k}^{b}=\sqrt{\frac{2 E_{k}^{0}}{j m^{b}}},
$$

where $E_{k}^{0}$ is the full energy component of the neutral beam source $k$, and $m^{b}$ is the mass of the beam neutral. With the assumption that the poloidal velocity is small compared to the beam velocity, $v_{\theta}<<v_{b}$, the collision velocity is given by,

$$
\left|\vec{v}-\vec{v}_{j k}^{b}\right|^{2}=\left(v_{\|}+v_{\phi}\right)^{2}+v_{\perp}^{2}+\left(v_{j k}^{b}\right)^{2}-2\left(v_{\|}+v_{\phi}\right) v_{j k}^{b} \sin \gamma_{k}+2 v_{\perp} v_{j k}^{b} \cos \gamma_{k} \sin \theta_{g}
$$

$v_{\phi}$ can have a significant effect on the reaction rate, even without a toroidal component to the view. The last two terms in Eq. (9) are dependent on the viewing geometry (with respect to the neutral beam); the last term is dependent on the gyro position where the charge exchange event took place. Integrating Eq. (4) over time gives

$$
\vec{v}_{a p p}^{ \pm}=V_{\phi} \hat{\phi}+\left( \pm v_{\theta} \sin \beta+\frac{V_{r}}{1+\omega^{2} \tau^{2}}\right) \hat{r}-\left(v_{\theta} \cos \beta+\frac{\omega \tau V_{r}}{1+\omega^{2} \tau^{2}}\right) \hat{z}
$$

with the definitions,

$$
\begin{aligned}
V_{\phi} \equiv & \frac{\sum_{j} \sum_{k} \iiint d v^{3} n_{j k}^{b} n^{i} \sigma_{j k}^{C X}\left|\vec{v}-\vec{v}_{j k}^{b}\right| f\left(\vec{v}-\vec{v}_{r o t}\right)\left(v_{\|}+v_{\varphi}\right)}{\sum_{j} \sum_{k} \iiint d v^{3} n_{j k}^{b} n^{i} \sigma_{j k}^{C X}\left|\vec{v}-\vec{v}_{j k}^{b}\right| f\left(\vec{v}-\vec{v}_{r o t}\right)}, \\
V_{r} \equiv & \frac{\sum_{j} \sum_{k} \iiint d v^{3} n_{j k}^{b} n^{i} \sigma_{j k}^{C X}\left|\vec{v}-\vec{v}_{j k}^{b}\right| f\left(\vec{v}-\vec{v}_{r o t}\right) v_{r}}{\sum_{j} \sum_{k} \iiint d v^{3} n_{j k}^{b} n^{i} \sigma_{j k}^{C X}\left|\vec{v}-\vec{v}_{j k}^{b}\right| f\left(\vec{v}-\vec{v}_{r o t}\right)},
\end{aligned}
$$

where $v_{r}=v_{\perp} \sin \theta_{g}$. Note that the corresponding term $V_{z}=0 . V_{\phi}$ and $V_{r}$ are the pseudo velocities associated with the charge exchange cross section that would be expected without gyro motion; these terms are evaluated numerically. Defining unit vectors along the direction of the upper $(+)$ and lower $(-)$ sightlines (see Fig. 3c),

$$
\hat{s}^{ \pm}=\sin \alpha \hat{r} \mp \cos \alpha \hat{z},
$$


the observed velocities from the upper and lower sightlines are given by

$$
\hat{s}^{ \pm} \cdot \vec{v}_{a p p}^{ \pm}= \pm v_{\theta} \cos (\alpha-\beta)+\frac{V_{r}}{1+\omega^{2} \tau^{2}}(\sin \alpha \pm \omega \tau \cos \alpha)
$$

The first term is the component of the desired poloidal velocity (of the parent ion $\mathrm{C}^{6+}$ ) along the line of sight, and the remaining terms are pseudo velocities associated with the charge exchange cross section and the gyro-orbit finite-lifetime effects. Defining new symmetric and asymmetric terms to isolate vertical and radial components,

$$
\begin{gathered}
v_{\text {sym }}=\frac{1}{2}\left(\hat{s}^{+} \cdot \vec{v}_{\text {app }}^{+}+\hat{s}^{-} \cdot \vec{v}_{\text {app }}^{-}\right)=\frac{V_{r}}{1+\omega^{2} \tau^{2}} \sin \alpha, \\
v_{\text {asym }}=\frac{1}{2}\left(\hat{s}^{+} \cdot \vec{v}_{\text {app }}^{+}-\hat{s}^{-} \cdot \vec{v}_{\text {app }}^{-}\right)=v_{\theta} \cos (\alpha-\beta)+\frac{\omega \tau V_{r}}{1+\omega^{2} \tau^{2}} \cos \alpha .
\end{gathered}
$$

Equation (14) can be used to check the accuracy of the calculations of the charge exchange cross section against measurements, since this term does not depend on the poloidal velocity. The second term in Eq. (15) is the correction term necessary to recover the poloidal velocity. Properly evaluating it requires accurate charge exchange cross sections and an accurate knowledge of $\tau$, the lifetime of the observed state. Rearranging Eqs. (14-15), one can solve for the poloidal velocity,

$$
v_{\theta} \cos (\alpha-\beta)=v_{\text {asym }}-\frac{\omega \tau}{\tan \alpha} v_{\text {sym }}
$$

This demonstrates a possible alternative to evaluating $V_{r}$ from Eq. (11) by using up/down symmetric views while maintaining large vertical angles, $\alpha \approx \pi / 4$, for the sightlines. The complex integrals over velocity space that required detailed knowledge of the beam neutral density, the charge exchange cross section, the population of excited neutrals could instead be measured with $v_{\text {sym }}$. So, even though the lifetime would still have to be evaluated, the system is more manageable. Ironically, when ignoring gyro motion, it was thought desirable to keep the $\alpha=0$ to avoid complications from the charge exchange cross section. Instead, we have the most information with $\alpha$ large. When $v_{\theta}=0$, we can measure the lifetime directly,

$$
\tau=\frac{v_{\text {asym }}}{v_{\text {sym }}} \frac{\tan \alpha}{\omega} .
$$

When viewing through the magnetic axis, the condition $v_{\theta} \approx 0$ should be approximately true, even though the outward shift of the magnetic flux surfaces due to the plasma pressure (Shafranov shift) can result in some finite velocity being observed along a chord through the magnetic axis. This will allow a check of the lifetime of the observed state that can be compared to calculations to test the accuracy of the correction term in Eq. (15).

\section{EXPERIMENTAL MEASUREMENTS}

The TFTR tokamak has a major radius $R_{0}=2.65 \mathrm{~m}$ and a minor radius $a=0.95 \mathrm{~m}$. A toroidal magnetic field $B_{\phi}=4.5 \mathrm{~T}$ and plasma current $I_{p}=1.6 \mathrm{MA}$ were used for the data presented here. Three neutral beam sources with acceleration voltages of $90-95 \mathrm{kV}$ are viewed by the poloidal rotation diagnostic from above and below the midplane with symmetric views. The sightlines intersecting the beam volume have angles from vertical $-16.8^{\circ} \leq \alpha \leq 4.6^{\circ}$ with $0^{\circ}$ intersecting the midplane at $\mathrm{R}=$ $3.09 \mathrm{~m}$. The height of the beam volume in the plasma is $45 \mathrm{~cm}$ FWHM, so measurements of poloidal flow are chord-averaged. We will use $u$ to indicate chordaveraged values of velocity and $v$ to indicate local values. 


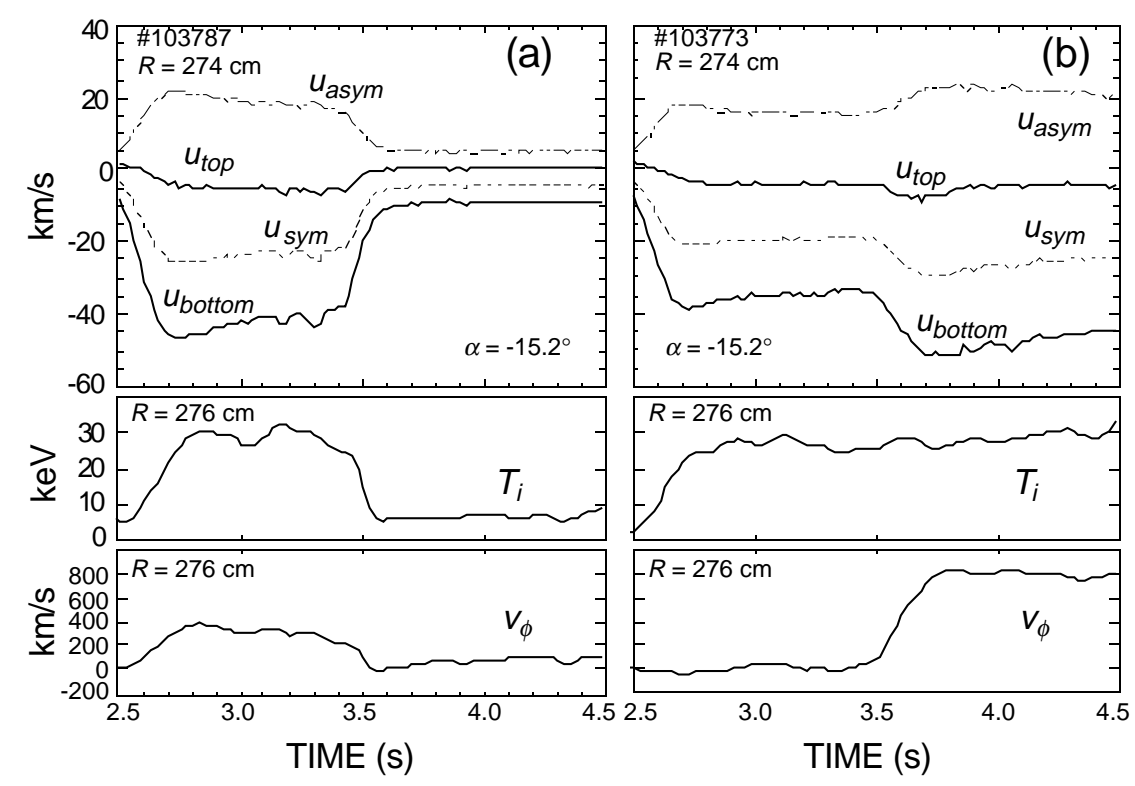

Figure 4. Two discharges illustrating the dependence of measured velocities on viewing direction, $T_{i}$, and $v_{\phi} . u_{\text {top }}$ and $u_{\text {bottom }}$ are the measured line shifts from the sightlines at the top and bottom of the TFTR tokamak, respectively. The derived velocity $u_{\text {sym }}=\left(u_{\text {top }}+u_{\text {bottom }}\right) / 2$ is purely the result of charge exchange effects; $u_{\text {asym }}=\left(u_{\text {top }}-u_{\text {bottom }}\right) / 2$ includes both poloidal flow and shifts due to gyro motion and finite lifetime effects

We examine two discharges in Fig. 4 with $14 \mathrm{MW}$ of deuterium neutral beam injection, central ion temperatures $T_{i}(0) \approx 30 \mathrm{keV}$, central electron density $n_{e}(0) \approx$ $5 \times 10^{13} \mathrm{~cm}^{-3}$ with $T_{e} \approx T_{i} / 3$. The measured velocity shift from the top of the machine is designated $u_{t o p}$, with the corresponding velocity viewed from the bottom $u_{\text {bottom }}$. The large differences in the magnitudes of $u_{\text {top }}$ and $u_{\text {bottom }}$ illustrate the strong view dependence of the gyro motion effects. Derived quantities that correspond to chord-averaged values of $v_{s y m}$ and $v_{a s y m}$ of Eqs. (14-15) are $u_{s y m}$ and $u_{a s y m}$, respectively. In the absence of gyro motion (or for $\omega \tau=0$ ), $u_{\text {asym }}$ would be the chordaveraged poloidal velocity. $u_{\text {sym }}$ corresponds solely to a line shift due to effects of the charge exchange cross section. For the discharge shown in Fig. 4a, the high $T_{i}$ established early is spoiled by puffing helium at 3.4-3.5 seconds reducing confinement. As $T_{i}$ decreases, there is a corresponding reduction is the measured velocities; the change in $u_{\text {sym }}$ demonstrates the dependence of the charge exchange shift on $T_{i}$. For the discharge in Fig. $4 \mathrm{~b}$, the neutral beams were changed from balanced injection to all co-directed injection at 3.5 seconds. Even though $T_{i}$ remains relatively constant, there is an increase in the measured velocities as $v_{\phi}$ increases from zero to $v_{\phi}=800 \mathrm{~km} / \mathrm{s}$. This large $v_{\phi}$ downshifts the collision velocity, reducing the reaction rate, and changing the local gradient in the reaction rate for each of the three beam energy components (see Fig. 1).

Initially, we will treat the $n=8$ level of carbon alone, i.e. no radiative cascades from other levels, and only collision processes (excitation and ionization) that de-populate the $n=8$ level. We will compute an effective charge exchange rate including ground state and $n_{D}=2$ excited beam neutrals. We compute local quantities using measurements of $T_{i}$, $T_{e}$, and $n_{e}$ mapped into the poloidal plane, and then determine a chord-averaged line shift to compare to measurements. A beam attenuation calculation based on a collisional radiative model by Boley, et al. 15 is performed to compute the local beam neutral density. The fractional populations of the neutral excited states relative to the ground state were evaluated using the same model. The atomic transition probabilities, compiled 
by Wiese et al. ${ }^{16}$, assume a statistical population between $l$ levels within an $n$ level. Excitation and ionization rates from electron impact were taken from Sampson and Zhang. ${ }^{17}$ Excitation and ionization rates from ion impact were calculated using the Classical Trajectory Monte Carlo (CTMC) code of Olson. ${ }^{18}$

Using the recommended cross sections for electron capture into the $n=8$ level of $\mathrm{C}^{5+}$ from Janev et al., 19 the calculated value of $u_{\text {sym }}$ for a central chord is overestimated, particularly during the period with high $v_{\phi}$ (see Fig. 5a). This suggests that the gradient in the total effective charge exchange reaction rate $\langle\sigma v\rangle$ eff used (with both ground state and excited beam neutrals) is too steep in the regions where the three beam components are sampling it. At higher $v_{\phi}$, the collision energies of the three beam components are shifted to lower collision energies (see Fig. 5b). The larger difference between calculated and measured values at high $v_{\phi}$ indicates that the gradient in $\langle\sigma v\rangle$ eff should be much smaller at lower collision energies. The cross sections used are recommended for collision energies above $20 \mathrm{keV} / \mathrm{amu}$ for $n$ levels away from the critical level $\left(n=4\right.$ for $\left.n_{D}=1\right)$. For comparison, we show an identical calculation using the capture cross section from ADAS, 20 taken from Fig. 3 of Ref. 7, which has a smaller gradient, especially at lower collision energies (Fig. 5a). The agreement with the measured $u_{s y m}$ is quite good for low $v_{\phi}$, but again there is a large discrepancy as the $v_{\phi}$ increases. The differences in these published cross sections with uncertainties of $20-25 \%$ illustrate some of the issues in relying on the details of the cross sections, particularly at low collision energies. At the same time, it is encouraging that this simple treatment can capture the dynamics for the balanced injection case, and perhaps we need only consider further atomic processes relevant to the lower collision energy range to improve overall agreement. One possibility is the inclusion of further excited levels for the beam neutrals, which are important at low collision energies despite their sparse populations. Figure 6 shows the charge exchange cross sections for beam neutrals in the first three states with $\mathrm{C}^{6+}$. The predominant level populated at low collision energy for $n_{D}=2$ is the upper level we observe, $n=8$. For charge exchange with $n_{D}=3$ beam neutrals, the predominant $\mathrm{C}^{5+}$ level populated is $n=12$. The excited populations for

(a)

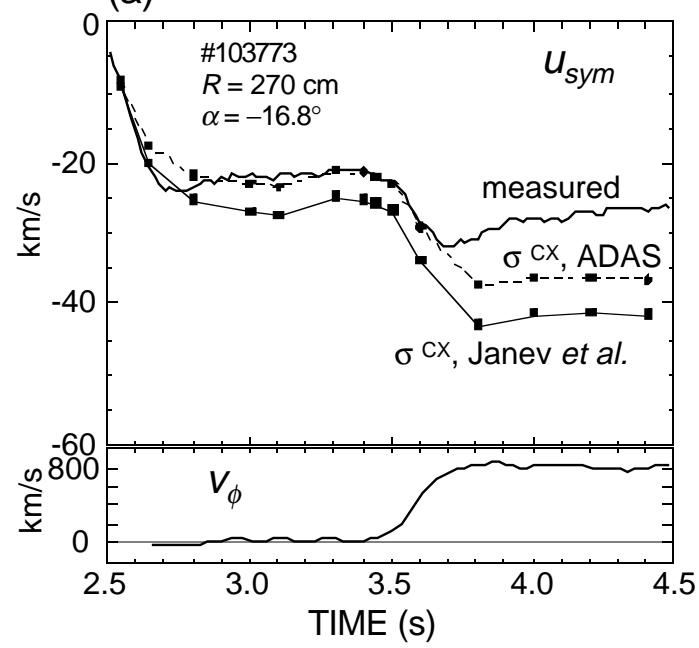

(b)

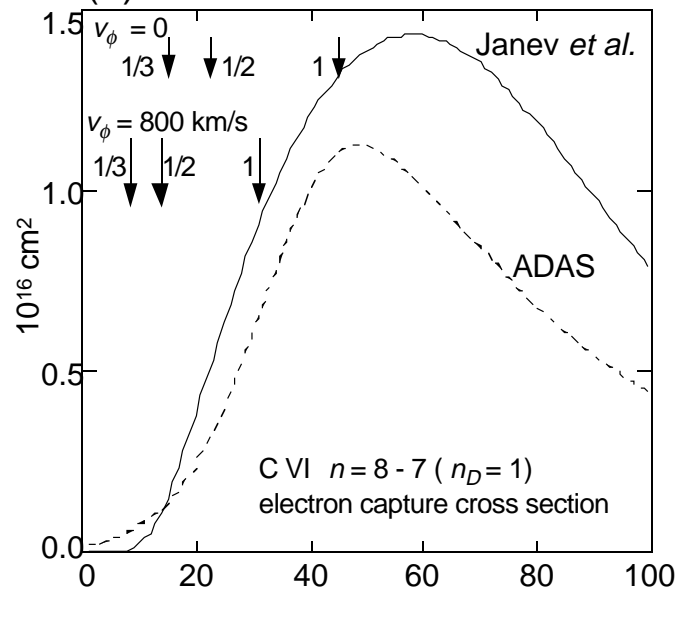

Figure 5. (a) Comparison of calculated to measured values of $u_{s y m}$, considering only $n=8$ level of carbon. Agreement worsens as toroidal velocity increases. (b) Electron capture cross sections for C VI $n=8-7\left(n_{D}=1\right)$ as recommended by Janev et al. (Ref. 19), and from ADAS (Ref 20). Arrows indicate center of distributions for full, half, and third energy components at zero toroidal velocity and the down shift in collision energy for $v_{\phi}=800 \mathrm{~km} / \mathrm{s}$. 


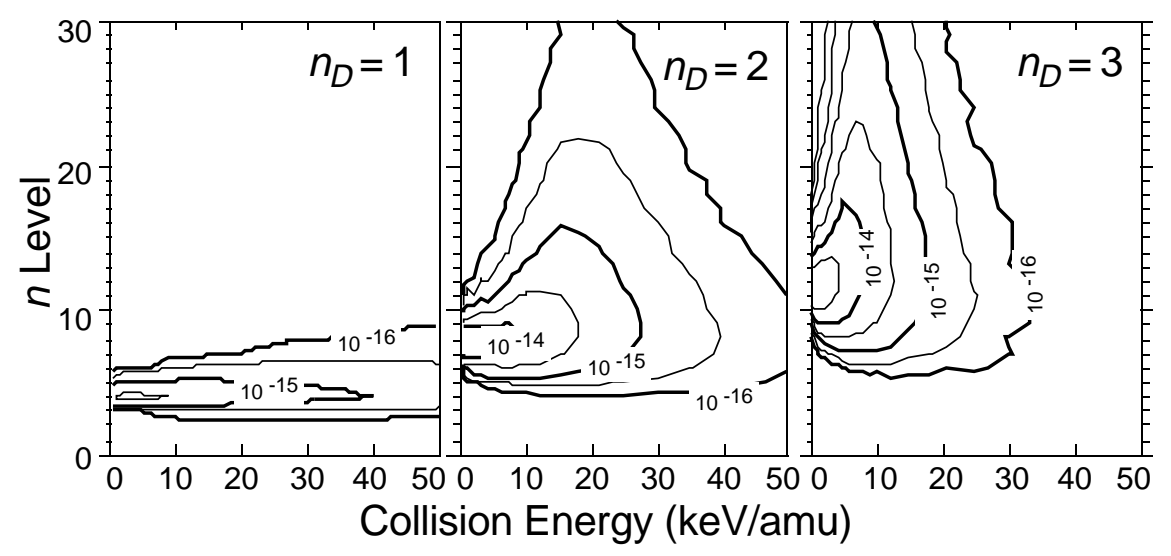

Figure 6. Electron capture cross sections (contours in units of $\mathrm{cm}^{2}$ ) into $\mathrm{C}^{5+}$ from deuterium beam neutrals in the ground state, $n_{D}=1$ (Ref. 19) and excited levels, $n_{D}=2,3$ (CTMC calculations).

$n_{D}=3$ are somewhat less than for $n_{D}=2$, but the cross sections (near $n=12$ ) are higher. Radiative cascades need to be included in our calculation to consider these higher $n$ levels. The higher $n$ levels also have longer lifetimes, so radiative cascades will extend the lifetime of the population for the $n=8$ level, and increase the contribution to the apparent velocity from the gyro motion.

A number of observed transitions of $\mathrm{C}^{5+}$ observed on TFTR are shown in Table. 1, demonstrating significant populations for $n$ levels much above $n=8$. The $n=17-11$ transition is routinely observed at the start of neutral beam injection before significant plasma heating has blended it with the $n=8-7$ line. The other lines were observed prior to the use of a spectral bandpass filter on the poloidal rotation diagnostic.

\begin{tabular}{cc} 
TABLE 1. Observed C VI Charge Exchange Lines \\
\hline n' - n & Wavelength $(\stackrel{\AA}{\mathbf{A}})$ \\
\hline $8-7$ & 5290.5 \\
$14-10$ & 5166.7 \\
$17-11$ & 5267.5 \\
$22-12$ & 5187.5 \\
\hline
\end{tabular}

\section{COLLISIONAL-RADIATIVE MODEL}

A collisional-radiative model is used to determine the population of the observed state following charge exchange which is affected by ionization, excitation, deexitation, and radiative cascades from other levels. Here we consider $n$ levels from 1-20 for $\mathrm{C}^{5+}$, but we do not resolve the individual $l$ levels. We solve a set of differential rate equations,

$$
\frac{d N_{i}}{d t}=S_{i}+M_{i j} N_{j}
$$

where $N i$ is the population of $n$ level $i, S_{i}$ is the source rate. $M_{i j}$ is a rate matrix defined

$$
M_{i j}=A_{i j}+n_{e} Q_{i j}-\delta_{i j}\left[\sum_{k}\left(A_{i k}+n_{e} Q_{i k}\right)+n_{e} Q_{i}\right],
$$

where $A i j$ are the transition probabilities from level $i$ to level $j, n_{e}$ is the electron density, $Q_{i j}=Q_{i j}^{\text {electron }}+Q_{i j}^{i o n}$ is the total of the excitation/deexcitation coefficients from level $i$ to $j$, from both ions and electron collisions, and $Q_{i}=Q_{i}^{\text {electron }}+Q_{i}^{\text {ion }}$ is the total ionization 
coefficient out of level $i$. The first term represents cascades from level $i$ into level $j$, the second, excitation when $i<j$ and deexcitation when $i>j$. The diagonal elements of the matrix ( $\delta_{i j}$ is the delta function) contain the sum of the loss terms. For evaluating the population produced by charge exchange, we take $S_{i}=0$ and set the initial values of $N_{i}$ according to the relative charge exchange rates for particular plasma parameters.

We no longer assume an exponential decay of the population in the level of interest and will calculate rates as a function of position on the gyro orbit. It is convenient to use cylindrical coordinates, recognizing that the angular coordinate in velocity space is identical to the gyro angle. The analogs of Eqs. (14-15) can be written

$$
\begin{gathered}
v_{\text {sym }}=\sin \alpha\left[\frac{\int P\left[A \sin \theta_{g}-B \cos \theta_{g}\right] \delta\left(\theta_{v}-\theta_{g}\right) d \theta_{v}}{\int R C \delta\left(\theta_{v}-\theta_{g}\right) d \theta_{v}}\right]=\sin \alpha\left[\frac{\int P A \sin \theta_{g} d \theta_{g}}{\int R C d \theta_{g}}\right], \\
v_{\text {asym }}=v_{\theta} \cos (\alpha-\beta)+\cos \alpha\left[\frac{\int P B \sin \theta_{g} d \theta_{g}}{\int R C d \theta_{g}}\right] .
\end{gathered}
$$

The values $A, B, C$ are time integrals of the yet unknown function $F\left(\theta_{g}, t\right)$, which is the decay of the population in the observed $n$ level,

$$
\begin{aligned}
& A\left(\theta_{g}\right)=\int F\left(\theta_{g}, \mathrm{t}\right) \cos \omega t d t, \\
& B\left(\theta_{g}\right)=\int F\left(\theta_{g}, \mathrm{t}\right) \sin \omega t d t, \\
& C\left(\theta_{g}\right)=\int F\left(\theta_{g}, \mathrm{t}\right) d t .
\end{aligned}
$$

$F\left(\theta_{g}, t\right)$ has a weak dependence on gyro angle and its time history will be determined by the collisional-radiative model. The values $R\left(\theta_{g}\right)$ and $P\left(\theta_{g}\right)$ refer to rates and perpendicular velocity weighted rates of the level of interest $(n=8)$.

$$
P\left(\theta_{g}\right)=\sum_{j} \sum_{k} \iint n_{j k}^{b} n^{i} \sigma_{8 j k}^{C X}\left|\vec{v}-\vec{v}_{j k}^{b}\right| f\left(\vec{v}-\vec{v}_{r o t}\right) v_{\perp}^{2} d v_{\phi} d v_{\perp}
$$

The rates are computed for all $n$ levels,

$$
R_{n}\left(\theta_{g}\right)=\sum_{j} \sum_{k} \iint n_{j k}^{b} n^{i} \sigma_{n j k}^{C X}\left|\vec{v}-\vec{v}_{j k}^{b}\right| f\left(\vec{v}-\vec{v}_{r o t}\right) v_{\perp} d v_{\phi} d v_{\perp}
$$

where $\sigma_{n j k}^{C X}$ is the total charge exchange cross section for level $n$. The charge exchange cross sections used here are from recommended cross sections by Janev et al. for $n=2-8$ which are extrapolated to $n=20$ assuming an exponential dependence with $n$ as suggested by Fritsch. 21

The initial condition for Eq. (18) uses populations proportional to the charge exchange rates for each level,

$$
N_{i}\left(\theta_{g}, t=0\right) \propto R_{i}\left(\theta_{g}\right) .
$$

After solving the rate equations (18) for $N_{i}(t)$, the function $F$ is then given by

$$
F\left(\theta_{g}, \mathrm{t}\right)=\frac{N_{8}\left(\theta_{g}, t\right)}{N_{8}\left(\theta_{g}, t=0\right)},
$$

where $N_{8}$ is the population of the upper level $n=8$ of our observed transition for carbon. With collisions and cascades, the decay of the observed state is no longer exponential, but we can define an effective lifetime, 


$$
\tau_{\text {eff }}=\frac{\int F\left(\theta_{g}, t\right) t d t}{\int F\left(\theta_{g}, t\right) d t} .
$$

When $v_{\theta}=0$, we can make an approximate measure of the effective lifetime assuming the dependence of $F\left(\theta_{g}, t\right)$ on gyro angle is small (calculations show variations of a few percent),

$$
\omega \tau_{\text {eff }}=\tan \alpha \frac{v_{\text {asym }}}{v_{\text {sym }}} \cong \frac{\bar{B}}{\bar{A}}=\frac{\int B d \theta_{g}}{\int A d \theta_{g}},
$$

where the bar indicates an average over gyro angle. When $F\left(\theta_{g}, t\right)=\mathrm{e}^{-\mathrm{t} / \tau}$, this reduces to Eq. (17). Using Eqs. (20-21) with Eq. (28), we solve for the poloidal velocity term,

$$
v_{\theta} \cos (\alpha-\beta) \approx v_{\text {asym }}-\frac{\bar{B} v_{\text {sym }}}{\bar{A} \tan \alpha}=v_{\text {asym }}-\frac{\omega \tau_{\text {eff }}}{\tan \alpha} v_{\text {sym }},
$$

which is the analog to Eq. (16).

Figure 7 shows a comparison of the calculated $u_{s y m}$ with the measured value for a central sightline using the collisional-radiative model. Again, the calculated value overestimates the measured value; the discrepancy increases with $v_{\phi}$. This more complete treatment indicates that the gradient in $\langle\sigma v\rangle$ eff is still too steep, especially at low collision energies. We can artificially increase the relative populations of $n_{D}=2,3$ to reduce the gradient in $\langle\sigma v\rangle$ eff; increasing the excited populations by a factor of 3.2 (open symbols, Fig. 7), give reasonable agreement with measured values of $u_{s y m}$, even at high $v_{\phi}$. There are several possible reasons why the computed $\langle\sigma v\rangle$ eff may be too steep. The charge exchange cross sections used may be inaccurate, especially for the lower collision energies. The estimated populations of the excited states may be underestimated. The presence of halo neutrals ${ }^{22,23}$ may also be influencing the gradient in $\langle\sigma v\rangle$ eff. Halo neutrals are produced by charge exchange of beam neutrals with

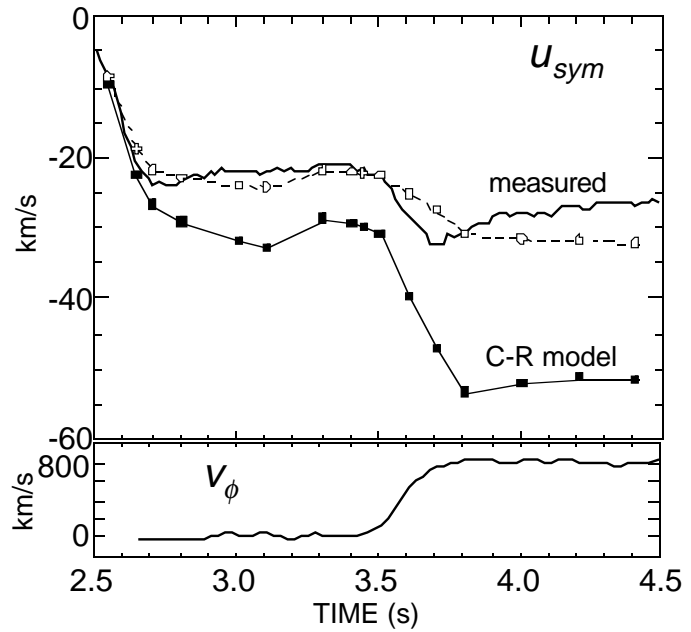

Figure 7. Comparison of calculated values of $u_{\text {sym }}$ with the collisional radiative model. The dashed line uses the CR model with the $n_{D}=2,3$ populations increased by a factor of 3.2 above estimates. plasma deuterons. The halo population has been calculated to be comparable to the beam neutral density. 22,23 The cloud of halo neutrals with thermal energy surrounds the beam volume and provides a source of neutrals that can charge exchange with the $\mathrm{C}^{6+}$ ions. Halo neutrals, unlike the energetic beam neutrals, are relatively isotropic, so while they contribute to the reaction rate they will not exhibit any variation over the gyro orbit, diluting the effect of the highly directional beam neutrals. For the high core $T_{i}$ in TFTR, the halo neutrals will interact with the charge exchange cross section at the low and intermediate collision energies, flattening the gradient in $\langle\sigma v\rangle$ eff where it now seems too steep. The halo neutral population needs to be evaluated to accurately assess its contribution is to the total charge exchange reaction rate. 


\section{LIFETIME EVALUATION}

The correction term needed to obtain the correct $v_{\phi}$ is proportional to $\omega \tau$, so evaluation of the correct lifetime of the observed levels is necessary. When evaluating the $n=8$ level by itself, we assumed an exponential decay of the population in that level. The decay rate used was the vacuum radiative lifetime $(0.54 \mathrm{~ns}$ for C VI $n=8$ 7) which was further reduced by depopulating collisions. In the collisional-radiative model, we allowed radiative decay from higher $n$ levels yielding longer non-exponential decay (Fig. 8). Estimated lifetimes from the collisional-radiative model were $\tau_{\text {eff }} \approx 0.7 \mathrm{~ns}$. A measurement of the lifetime can also be made by assuming that the chord-averaged poloidal rotation from a sightline through the magnetic axis is approximately zero and using Eqs. (17) or (28). The experimentally derived effective lifetime (Fig. 8) is much longer than the lifetime of the collisional-radiative model, $\tau_{\text {eff }} \approx 1.1 \mathrm{~ns}$. The transition probabilities used to determine the lifetimes assumed that the $l$ levels within an $n$ level maintain a statistical population, i.e. the $l$ levels were fully mixed. Using collisional cross sections for $T_{i}=5 \mathrm{keV}$ from Olson, ${ }^{24}$ we calculate mixing rates and estimate the effective lifetime of the $n=8$ level without radiative cascades as a function of ion density (see Fig. 9). For the range of densities in our discharge, the effective lifetime of the $n=8$ level doubles to near the experimentally estimated values shown in Fig. 7. A complete treatment would require $n, l$ resolved charge exchange cross sections and mixing rates for the $n$ levels in the model.

\section{SUMMARY}

We have shown that poloidal rotation measurements with charge exchange from neutral beams are complicated by extraneous line shifts due to the gyro motion of the ion and its finite lifetime. This effect scales with $T_{i}$ and $B_{\phi}$. A collisional radiative model, using recommended reaction rates, was used to estimate the pseudo velocity due to the charge exchange effects, which was compared to measurements. The model overestimated the effect of the gyro motion and underestimated the effective lifetime of the observed state. A large halo neutral population is a possible explanation for the overestimate of the gyro orbit effect in the calculation and should be explored. The large difference between the calculated and measured effective lifetime suggests that $l$ levels
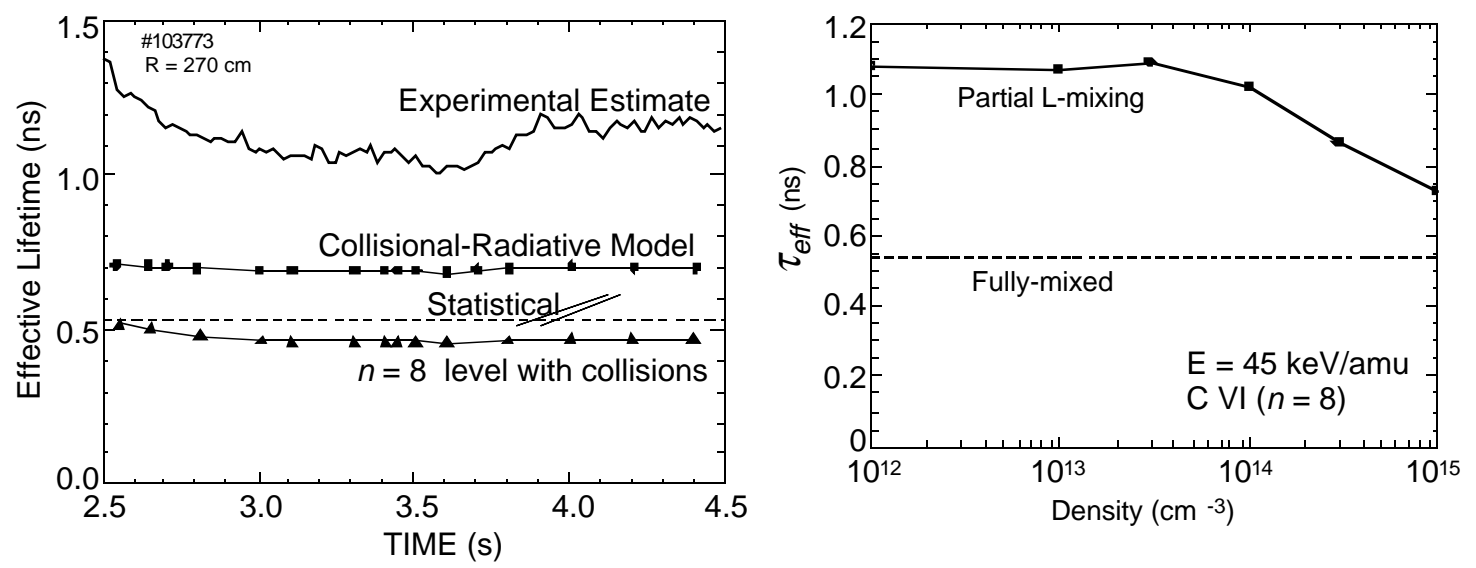

Figure 8. Estimated effective lifetimes of the $n=8$ Figure 9. Effect of $l$ mixing on the effective level of $\mathrm{C}^{5+}$ for a sightline passing near the lifetime of the CVI $n=8$ level over a range of magnetic axis. ion densities. 
for $n=8$ are relatively unmixed for the plasmas measured. An experimental estimate of the lifetime of the $n=8$ level of $\mathrm{C}^{5+}$ is about $1.1 \mathrm{~ns}$. Poloidal rotation measurements with symmetric views at large angles to the neutral beam direction may well avoid the complicated atomic physics necessary to calculate corrections to the apparent $v_{\theta}$.

\section{ACKNOWLEDGMENTS}

The authors would like to acknowledge discussions with Dr. M. C. Zarnstorff who first pointed out the importance of the gyro motion and finite lifetime on the interpretation of the poloidal rotation measurements. The authors would also like to thank Dr. R. E. Olson for making his CTMC code available for computation of cross sections necessary for this study. This work was supported by U. S. Dept. of Energy contract DE-AC02-76-CH03073.

\section{REFERENCES}

1. R. C. Isler, Plasma Phys. Control. Fusion 36, 171-208 (1994), and references therein.

2. R. J. Groebner, N. H. Brooks, K. H. Burrell, L. Rottle, Appl. Phys. Lett. 43, 920 (1983).

3. R. E. Bell, F. M. Levinton, S. H. Batha, E. J. Synakowski, and M. C. Zarnstorff, Phys Rev. Lett. 81, 1429-1432 (1998).

4. R. B. Howell, R. J. Fonck, R. J. Knize, K. P. Jaehnig, Rev. Sci. Instrum. 59, 1521-1523 (1988).

5. M. von Hellermann, P. Breger, J. Frieling, R. König, W. Mandl, A. Maas, H. P. Summers, Plasma Phys. Control. Fusion 37, 71-94 (1995).

6. R. C. Isler, R. E. Olson, Phys. Rev A, 37, 3399-3402 (1988).

7. R. Hoekstra, H. Anderson, F. W. Bliek, M. von Hellermann, C.F.Maggi, R. E. Olson, H. P. Summers, Plasma Phys. Control. Fusion 40, 1541-1550 (1998).

8. R. E. Bell, Rev. Sci. Instrum., 68, 1273-1280 (1997).

9. R. E. Bell, L. E. Dudek, B. Grek, D. W. Johnson, R. W. Palladino, Rev. Sci. Instrum. 70, 821828, (1998).

10. F. M. Levinton, R. E. Bell, S. H. Batha, E. J. Synakowski, M. C. Zarnstorff, Phys. Rev. Lett. 80, 4887 (1998).

11. M. C. Zarnstorff, [private communication].

12. A. R. Field, G. Fussmann, J. V. Hofmann, and the ASDEX Team, Nucl. Fusion, 32, 1191-1208 (1992).

13. D. D. Ryutov, Phys. Plasmas 7, 1315-1320 (2000).

14. A. Boileau, M. von Hellermann, L. D. Horton, J. Spence, H. P. Summers, Plasma Phys. Control. Fusion 31, 779-804 (1989).

15. C. D. Boley, R. K. Janev, D. E. Post, Phys. Rev. Lett. 52, 534-537 (1984).

16. W. L. Wiese, M. W. Smith, B. M. Glennon, "Atomic Transition Probabilities, Volume I Hydrogen Through Neon", NSRDS-NBS 4, National Standard Reference Data Series, National Bureau of Standards 4, U. S. Government Printing Office, Washington, D. C., (1966).

17. D. H. Sampson, H. L. Zhang, Astrophy. J., 335, 516-524 (1988).

18. R. E. Olson, Phys. Rev. A, 24, 1726-1733 (1981).

19. R. K. Janev, R. A. Phaneuf, H. Tawara, T. Shirai, At. Data Nucl. Data Tables, 55, 201-232 (1993).

20. H. P. Summers, "Atomic Data and Analysis Structure", JET Joint Undertaking Report JET-IR(94)-06, http://patiala.phys.strath.ac.uk/adas

21. W. Fritsch, Phys. Rev. A, 30, 3324-3327 (1984).

22. B. C. Stratton, R. J. Fonck, A. .T. Ramsey, E. J. Synakowski, B. Grek, K. W. Hill, D. W. Johnson, D. K. Mansfield, H. Park, G. Taylor, P. M. Valanju, Nucl. Fusion 30, 675-687 (1990).

23. E. Busche, H. Euringer, R. Jaspers, Plasma Phys. control. Fusion 39, 1327-1338 (1997).

24. R. E. Olson, [private communication]. 
The Princeton Plasma Physics Laboratory is operated by Princeton University under contract with the U.S. Department of Energy.

\author{
Information Services \\ Princeton Plasma Physics Laboratory \\ P.O. Box 451 \\ Princeton, NJ 08543
}

Phone: 609-243-2750

Fax: 609-243-2751

e-mail: pppl_info@pppl.gov

Internet Address: http://www.pppl.gov 